\title{
AS CONTRIBUIÇÕES DE MAURÍCIO PEIXOTO PARA A MATEMÁTICA
} BRASILEIRA

\author{
MAURICIO PEIXOTO'S CONTRIBUTIONS TO BRAZILIAN \\ MATHEMATICS
}

\author{
Dianara Figueirêdo Freire ${ }^{1}$ \\ Universidade Estadual do Ceará - UECE \\ Antonio José Melo de Queiroz ${ }^{2}$ \\ Universidade Estadual do Ceará - UECE
}

\section{Resumo}

O desenvolvimento da ciência foi surgindo com o avançar dos tempos, foi assim em todo mundo. É importante ressaltar que em alguns lugares ela avançou mais depressa, porém no Brasil teve suas complicações, pois ainda no século XIX e início do século XX a nossa sociedade, até aquele momento, não se importava com o avanço da ciência. Desta maneira, não foi diferente com a Matemática, que teve várias tentativas, a fim de ter o avanço desejado no Brasil, contudo não obtiveram êxito até o inicio do século XX. A Matemática começou a ter seus primeiros progressos em pesquisa científica a partir da década de 1930, com isso, foram surgindo pesquisadores nas suas diversas áreas e é importante que a sociedade saiba quem foram esses pioneiros, pois assim saberemos o caminho trilhado por eles e seus respectivos avanços na Matemática. Sendo assim, esse artigo teve como objetivo analisar como se construiu a carreira de Maurício Matos Peixoto e verificar quais foram as principais contribuições dele para a matemática no Brasil. O trabalho foi desenvolvido por meio de uma pesquisa bibliográfica e documental de caráter exploratório. Verificamos que Maurício Peixoto foi um grande matemático que contribuiu principalmente para a área de Sistemas Dinâmicos, com ênfase em estabilidade estrutural. Trabalhou em várias instituições de cunho científico, como o Conselho Nacional de Desenvolvimento Científico e Tecnológico (CNPq) e a Academia Brasileira de Ciências (ABC), além colaborar na implantação e crescimento do Instituto de Matemática Pura e Aplicada (IMPA) e, portanto, contribuindo no crescimento da pesquisa Matemática no país. Dessa maneira, é perceptível o trabalho de Maurício Peixoto para a divulgação da Matemática. Assim, salientamos a relevância deste artigo para a nossa formação, pois é essencial sabermos quem fez parte da nossa história e mostrar a sociedade que existem brasileiros fazendo Matemática de alta qualidade.

Palavras-chave: Maurício Matos Peixoto; Estabilidade Estrutural; História da Matemática no Brasil.

\footnotetext{
1 dianara.freire@aluno.uece.br.

2antonio.queiroz@uece.br. 


\begin{abstract}
The development of Science was borning through all over the world. It has happened with the advance of the time. It is important to emphasize that in some places, it has advanced faster than the others one. However, there were some complications in Brazil because during the $19^{\text {th }}$ century and $20^{\text {th }}$ century, the Brazilian society didn't care with the development of Science. At this way, it wasn't different with Mathematics. They've tried a lot of times to advance in the beginning of $20^{\text {th }}$ century, but it was unsuccessful. The subject Mathematics had to start its first steps in scientific research from the 30's decades. With that, it was borning some researchers in different areas of studying. Besides that, it is important that all over world should know who they are and their contributions to the advance of Mathematics. Then, this article focus on analyze Mauricio Matos Peixoto's career and how it was constructed during the time. By the way, we are going to verify his contributions to Mathematics in Brazil. This research was developed based on a bibliographic research and documental exploratory. We have realized that Mauricio Peixoto was a big mathematician, who contributed, mainly, to the area in Dynamics System focusing on structural stability. He worked in several scientific institutions as the National Council for Scientific and Technological Development, Brazilian Academic of Science. All of this environments are helping to the growing of the Institute of Applied and Pure Mathematics and also, to contribute to the math research in our country. Thus, it is perceptible the contributions of that mathematician to the scientific divulgation. So, we claim the importance of this work to our formation because it is logical to understand who participated of our history. By the way, with this research, we can show to the society that exist Brazilian people who are able to create Mathematics in a high level.
\end{abstract}

Keywords: Mauricio Matos Peixoto; Structural Stability; Mathematic History in Brazil.

\title{
Introdução
}

O desenvolvimento da ciência no Brasil foi tardia. Provalvelmente, isso se deu por causa da nossa colonização ter sido por Portugal, um país retrógrado em relação a promoção da pesquisa científica. Deste modo, ainda no século XIX e início do século XX a nossa sociedade, até aquele momento, não se importava com o avanço da ciência, visto que não tinha, por parte dos governantes, o interesse de concientizar a população de que o país necessitava de pesquisas originais e de espaços para divulgação delas.

Não foi diferente com a Matemática, em que foram apresentadas várias tentativas para criação de cursos de ciências a fim do avanço desejado no Brasil, porém essas não obtiveram êxito até o início do século XX. Desta forma, o lugar mais acessível para estudar essa disciplina eram em cadeiras do curso de engenharia, pois os cursos de Matemática não haviam se expadindo no país. Portanto o retardo da Matemática em relação a outros paises foi inevitável. 
A Matemática começou a ter seus primeiros progressos em pesquisa científica a partir da década de 1930, com a criação da Universidade de São Paulo (USP), a qual tinha na sua composição a Faculdade de Filosofia, Ciências e Letras (FFCL-USP) que continha o curso de Matemática. Além disso, em 1937, foi fundada a Universidade do Brasil, no Rio de Janeiro, onde havia a Faculdade Nacional de Filosofia (FNFi) que foi a responsável por iniciar a pesquisa matemática nessa cidade.

Essas instituições trouxeram professores renomados do exterior para divulgação e ensino dessa disciplina, um dos professores que veio para USP, em 1934, foi Luigi Fantapié, de origem italiana. Seus estudos tinham ênfase em Análise Matemática. Sobre a USP, Silva (2003, p. 52), destaca: “[...] essa instituição foi o berço da atual Matemática brasileira em virtude dos estudos ali desenvolvidos a partir de 1934." A respeito da FNFi, Silva (2003, p. 52) comenta: "Nessa unidade foi criado o curso de Matemática a partir do qual floresceram os estudos matemáticos na cidade do Rio de Janeiro."

A partir de então, foram surgindo outras instituições, uma delas foi o Instituto de Matemática Pura e Aplicada (IMPA), criado em 1952. Os seus objetivos principais eram: "o ensino e a investigação científica no campo da Matemática pura e aplicada, assim como a difusão e elevação da cultura matemática no país" (CNPq, arquivo t.6.3.002 Apud SILVA, 2009, p. 274). Seus primeiros pesquisadores foram Lélio Gama (diretor), Leopoldo Nachbin e Maurício Matos Peixoto.

Esses órgãos contaram com pessoas que começaram a expandir a Matemática, através de pesquisas, seminários e criação de revistas científicas. Desta maneira, ela começou a ganhar espaço no contexto brasileiro, sendo criadas bolsas para o incentivo da pesquisa científica, bem como a implantação de bibliotecas, com a finalidade de melhorar o acesso ao conhecimento para pessoas interessadas nesta ciência. Assim, pesquisadores foram surgindo nas diversas áreas da Matemática.

É importante que a sociedade saiba quem foram esses pioneiros, pois assim saberemos o caminho trilhado por eles e seus respectivos avanços na Matemática, observando o crescimento da pesquisa científica nessa área e conhecendo como se deu sua evolução no Brasil até os dias atuais. Portanto é essencial estudos que mostrem os pesquisadores matemáticos brasileiros e suas contribuições para a difusão da Matemática no país. 
Deste modo, ao analisar nomes que foram importantes no desenvolvimento da Matemática no Brasil, apareceu o nome do pesquisador e professor Maurício Matos Peixoto, que como já mencionado acima foi um dos criadores do IMPA. Assim surgiu o seguinte questionamento que impulsionou essa pesquisa: Como se construiu a carreira de Maurício Matos Peixoto e em que ele contribuiu para a Matemática no Brasil?

Com base nesta indagação, esse artigo teve como objetivo analisar como se construiu a carreira de Maurício Matos Peixoto e verificar quais foram as principais contribuições dele para a Matemática no Brasil.

O trabalho foi desenvolvido por meio de uma pesquisa bibliográfica e documental de caráter exploratório, na qual buscamos saber do desenvolvimento da carreira de Maurício Peixoto, analisando suas contribuições para a Matemática e momentos que perpassaram a sua trajetória. A investigação foi por meio de artigos, entrevistas escritas e gravadas e também de livros como Silva (2008) que têm informações sobre o nosso objeto de pesquisa.

O referido artigo está dividido em quatro seções, na primeira se encontra a introdução com aspectos preliminares sobre o assunto, contando com a justificativa, questão e objetivo da pesquisa. O segundo tópico é intitulado por " $O$ início da jornada de Maurício Peixoto", e mostra as histórias que perpassaram o início da carreira desse matemático e professor.

A terceira parte, "A consolidação da carreira de matemático de Maurício Peixoto", conta com um breve histórico das contribuições que Mauricio Peixoto ofertou para a pesquisa científica na área da Matemática no Brasil e também divulga sua influência na difusão dessa disciplina no país. A quarta e última parte são as considerações finais, trazendo a colaboração do desenvolvimento deste trabalho.

\section{O início da jornada de Maurício Peixoto}

Maurício Matos Peixoto nasceu na cidade de Fortaleza, no dia 15 de abril de 1921, filho de José Carlos de Matos Peixoto e Violeta Rodrigues Peixoto. Seu Pai era governador do estado do Ceará, mas com a revolução de 1930 foi destituído do cargo e teve que se dirigir ao Rio de Janeiro com a família, que era composta por 8 filhos.

Todavia, em 1931, Maurício voltou ao Ceará para morar com uma tia e em 1932 terminou o primeiro ano do ginasial ainda em Fortaleza, retornando no final do ano para o Rio de Janeiro. Matriculou-se em 1933 no colégio Dom Pedro II com a finalidade de 
cursar o segundo ano do ginasial, onde reprovou, pois os seus estudos em Fortaleza foram deficientes.

Com a reprovação, conheceu o professor Nelson Chaves, que era aluno da Escola Nacional de Engenharia (ENE) e ficou encarregado de preparar Maurício Peixoto para o exame de segunda época, tal avaliação foi mencionada por ele em uma de suas entrevistas concedidas no ano de 2003. Nessa mesma, ele afirma que o professor foi sua inspiração para seguir uma carreira na área da Matemática. Sobre o docente ele alega que era,

Um jovem inteligente, com ideias muito claras sobre matemática. Foi uma referência muito importante, porque mudou a minha vida, num certo sentido. [...] e fiquei deslumbrado com suas aulas; nessa época decidi que iria estudar alguma coisa que envolvesse matemática (PEIXOTO, 2003, p. 240).

Podemos averiguar que Maurício na sua adolescência já havia decidido adentrar na área da Matemática. Ele foi aprovado no respectivo exame e passou seis anos no colégio Dom Pedro II. Quando saiu, passou um ano na Universidade do Distrito Federal (UDF) cursando Matemática, porém a mesma foi extinta.

Como na época haviam poucos cursos de Matemática no Brasil, ele ingressou na ENE da Universidade do Brasil, um lugar mais propício para quem queria estudar Matemática no país. Peixoto (2003, p. 240) afirma: “Como a carreira de matemático não existia, fui estudar a matéria no lugar onde era ensinada, ou seja, na Escola de Engenharia."

Nesse período, teve como colegas o professor e matemático Leopoldo Nachbin e a matemática Marília de Magalhães Chaves. Este primeiro, foi aluno da Escola Engenharia e se tornaram amigos. Sobre Leopoldo, Peixoto (2003, p. 241) informa: "Durante os cinco anos do curso de engenharia, Leopoldo e eu fomos companheiros inseparáveis."

Marília se tornou sua primeira esposa, ela participou do início da carreira matemática de Maurício, possuindo uma importante contribuição para o teorema conhecido como Peixoto e Peixoto. Peixoto (2003, p. 244) relata: "A influência de Marília foi muito grande durante esses "anos dourados"."

Em 1943, ele concluiu o curso de engenharia, porém nunca assumiu a profissão, mesmo tendo o cadastro do Conselho Regional de Engenharia e Arquitetura (CREA). Peixoto (2011) afirma, na sua entrevista aos Matemáticos Elon Lages e Enrique Pajuls, 
que nunca colocou nenhum tijolo em cima do outro como engenheiro. Deste modo, podemos perceber que, de fato, Peixoto entrou na ENE para estudar Matemática.

Sua desenvoltura com a área da Matemática era notável dentro da ENE e isso o levou a dar aulas particulares durante todo o curso juntamente com Leopoldo, pois eles já eram conhecidos pelo seu desenvolvimento nos estudos. Peixoto (2003) alega que se reuniam na biblioteca municipal e na Universidade para estudar assuntos referentes a Matemática, pois na segunda instituição havia um acervo de materiais significantes nessa área.

Sobre o destaque de Maurício, Silva discorreu,

O talento de Maurício Matos Peixoto para estudos da Matemática o levou, ainda como aluno da ENE, a assistir como ouvinte cursos ministrados na Faculdade Nacional de Filosofia-FNFi pelo Matemáticos italianos Luigi Sobrero e Gabriele Mammana (SILVA, 2008, p. 161).

No mesmo ano que saiu da escola de engenharia, entrou para ser professor assistente da mesma, ensinando a disciplina de Mecânica Racional, ainda sem receber, mas em seguida foi contratado. Fez o seu primeiro concurso para professor catedrático da Escola de Agronomia, foi aprovado, mas não foi indicado por nenhum dos cinco examinadores da banca.

Peixoto (2011) alega que não sabe o porquê, porém ouviu boatos que uma pessoa da banca disse que ele não havia sido indicado pois, por ser uma pessoa brilhante e competente, não teria dificuldades na sua carreira. Com isso, podemos notar que era perceptível a carreira promissora que Maurício Peixoto teria pela frente.

\section{A consolidação da carreira de matemático de Maurício Peixoto}

Com a conclusão do curso de engenharia em 1943, Peixoto tinha estudos com ênfase em Equações Diferenciais ligadas a Mecânica. Chegou a desenvolver tese para um concurso de professor catedrático ligado ao tema. Em 1947, participou do Núcleo de Matemática da Fundação Getúlio Vargas (FGV), no qual estava também Leopoldo Nachbin, Maria Laura Mousinho, Lélio Gama, entre outros.

Esse núcleo fornecia espaço para quem gostava de Matemática, nele tinham palestras, discussões de livros e foi onde desenvolveu-se a revista Summa Brasiliensis Mathematicae, que teve sua relevância na divulgação da Matemática investigada no Brasil. Sobre a revista, Peixoto (2003, p. 242) argumenta que "[...] publicou alguns 
artigos bastante importantes e sobreviveu ao núcleo da Fundação. [...] a Summa representou um marco importante na Matemática brasileira."

Apesar do núcleo ligado a FGV ter possuído duração de pouco mais de um ano, Peixoto conheceu matemáticos de renome, como Oscar Zariski, que veio para ministrar conferências. Posteriormente, em 1949, viajou para Chicago juntamente com Leopoldo, por meio de uma bolsa fornecida pela Convenção de Buenos Aires, a BA Convention, pois segundo Peixoto,

Vieram ao Rio dois importantes matemáticos americanos: Marshall Harvey Stone, analista, e Adrian Albert, algebrista. Viajavam como parte de um programa do Departamento de Estado, coisa como política de boa vizinhança, [...] Era uma bolsa do Departamento de Estado, que nos custou idas quase diárias ao Itamaraty durante cerca de um ano; o auxílio era da BA Convention, Convenção de Buenos Aires, através da qual o governo americano dava bolsas a latino-americanos (PEIXOTO, 2003, p. 243).

Ele retornou ao Brasil em 1951 com o intuito de fazer o concurso para professor catedrático da Universidade do Brasil. Peixoto desistiu de concluir o curso de doutorado no exterior para retornar ao país. É importante ressaltar que ele era doutor no Brasil, pois pela lei vigente na época, quem defendia tese recebia o título, e o mesmo havia feito isto, em 1948, para o concurso da Escola de Agronomia. Segundo ele era difícil o vínculo, sobre a abdicação do doutorado, Peixoto (2003, p. 243) afirma: "Nunca me arrependi de ter largado o doutorado, porque uma cátedra na Universidade do Brasil era muito difícil de aparecer, pois era vitalícia; não era oportunidade que desperdiçasse."

Em 1952 foi criado o IMPA e Maurício estava envolvido na sua fundação. Esta instituição foi marcante na vida dele, pois foi em sua biblioteca, folheando uma revista, que conheceu o seu objeto de estudo em um artigo intitulado: Sistemas Dinâmicos com Estrutura Estável, traduzido do russo por Henry DeBaggis. Sobre essa descoberta, Sotomayor expõe que

Como engenheiro, autodidata e professor catedrático de Mecânica Racional, com um conhecimento muito acima da média das equações diferenciais da Mecânica Clássica, Peixoto notou imediatamente que uma forma inteligente de estabilidade - ou continuidade - estava envolvida no texto do autor (SOTOMAYOR, 2019, p. 2, tradução nossa).

Ao saber que haveria uma conferência em Chicago e no evento estaria Solomon Lefschetz, um matemático já reconhecido por suas pesquisas na área, Peixoto (2011) alega que escreveu uma carta com um esbouço do que queria desenvolver sobre 
Estabilidade Estrutural, essa carta foi levada por Leopoldo, sendo retornada por Lefschetz com algumas considerações.

Desta forma, ele foi para a Universidade de Princeton em 1957, trabalhar como orientando de Lefschetz, com o tema: Estabilidade Estrutural de Equações Diferenciais, lá permaneceu por um ano. Assim, Peixoto iniciou os trabalhos nesta área em que tanto contribuiu. Sobre Solomon, Silva (2008, p. 163) afirma: "Maurício Matos Peixoto foi influenciado por este matemático. Posteriormente ele desenvolveu trabalhos pioneiros em Sistemas Dinâmicos e deu importantes contribuições nesta que é atualmente uma área de pesquisa muito vigorosa."

Um de seus trabalhos mais conhecidos foi o teorema de Peixoto que diz: $O s$ fluxos estruturalmente estáveis em superfícies são simplesmente caracterizados e constituem um aberto e denso no espaço de todos os fluxos (SILVA, 2008, p.163). Este foi pensado ainda no Brasil, em seminários ofertados na ENE. Sobre o seu feito, Peixoto relata que,

Comecei a pensar nessas coisas nuns seminários que promovíamos aos sábados [...]. Eu tinha feito uma demonstração inicial do teorema em 1957 e cheguei nos Estados Unidos com o negócio muito adiantado e ideias bastante claras. Afinal, eu já vinha estudando equações diferenciais e sistemas dinâmicos há algum tempo - estabilidade estrutural era a palavra-chave para isso (PEIXOTO, 2003, p. 244).

Portanto, observamos o quanto Peixoto se destacava na sua área de pesquisa, isso ajudou muito a Matemática brasileira, dando visibilidade. Além de pesquisas, ele desenvolveu seminários e trouxe importantes matemáticos ao IMPA, como Steve Smale, em 1960. A vinda desses matemáticos colaborou com o crescimento do instituto e da pesquisa Matemática brasileira. Sobre Smale, Peixoto declara:

\footnotetext{
Ele ficou muito interessado pelo meu trabalho. Sua bolsa em Princeton era de dois anos, e lhe foi permitido passar os últimos seis meses no Brasil, no IMPA que era um lugar praticamente desconhecido. [...] foi um trabalho muito importante, o que ele fez aqui. Deu visibilidade internacional ao IMPA. Nos primeiros anos do IMPA, a presença do Smale foi fundamental para sua consolidação (PEIXOTO, 2003, p. 245).
}

Maurício no decorrer da sua carreira participou de vários cargos em instituições de pesquisa, tendo passado pelo Conselho Nacional de Desenvolvimento Científico e Tecnológico (CNPq), sendo vice-presidente de 1971 a 1973, e também foi presidente da Academia Brasileira de Ciências (ABC) por dez anos. Contribuindo assim para o crescimento do conhecimento científico matemático do país. 
Peixoto se destacou bastante nas suas pesquisas. Dessa maneira, acerca do teorema de Peixoto e outros trabalhos que Maurício desenvolveu, Silva afirma que

Este e outros resultados também obtidos por ele e relacionados com o problema de contorno por dois pontos para equações ordinárias de segunda ordem, mais precisamente, relacionados com o problema de contar quantas soluções passam pelos dois pontos, fizeram de Maurício Matos Peixoto um dos matemáticos brasileiros contemporâneos de projeção internacional (SILVA, 2008, p. 164).

Ele escreveu muitas obras, entre artigos e livros, contribuindo assim para o reconhecimento da Matemática brasileira. Trabalhou como professor em outras universidades, como a USP, e em 1964 foi convidado por Lefschetz para ser professor da Brow University, nos Estados Unidos, aceitou o convite e ficou quatro anos fora do país. Segundo Peixoto (2003), o Brasil estava conturbado naquela época, foi bom passar esse tempo fora.

Com o seu crescimento nas pesquisas ele conquistou vários prêmios, um deles foi o prêmio Moinho Santista, em 1969, que é considerado um dos maiores prêmios brasileiros. Recebeu também, em 1987, o prêmio de Matemática da Academia de Ciências do Terceiro Mundo. Dessa maneira, podemos averiguar a grande contribuição que Maurício deu para a Matemática.

\section{Considerações finais}

Retornando ao objetivo deste trabalho, podemos observar o quanto Maurício Matos Peixoto contribuiu para a história dos Sistemas Dinâmicos, ele foi pioneiro da estabilidade estrutural. Desenvolveu vários trabalhos e deixou problemas que foram e estão sendo desenvolvidos por outros grandes matemáticos.

Esse trabalho contribuiu de forma eficaz para nossa formação, pois nos contemplou com uma parte, mesmo que pequena, da evolução Matemática no Brasil. Pois é extremamente importante que existam pesquisadores investigando o percurso desses professores e matemáticos para que nossa história seja constantemente relembrada e contada às várias gerações, mostrando a sociedade que existem brasileiros fazendo Matemática. 


\section{Referências}

LIMA, E. L; PUJALS. E. R. Entrevistas com Eméritos III - Elon Lages e Enrique Pujals entrevistam Maurício Peixoto, 2017, Rio de Janeiro. Disponível em: https://youtu.be/PToAegfcFKA. Acesso em: 20 de novembro de 2019.

PEIXOTO, M. M. Maurício Matos Peixoto. In: PALIS, J.; CAMACHO, C.; LIMA, E. L. (orgs.) IMPA 50 anos. Rio de Janeiro: IMPA, 2003. p. 240-250.

SILVA, C. M. O IMPA e a comunidade de matemáticos no Brasil. Cadernos de Pesquisa, v. 39, n. 138, p. 897-917, 2009.

SILVA, C. P. A matemática no Brasil: história do seu desenvolvimento. 3. ed. rev. São Paulo: Blucher, 2003.

SILVA, C. P. Início e consolidação da pesquisa matemática no Brasil. Brasília: Senado Federal, Conselho Editorial, 2008.

SOTOMAYOR, Jorge. On Maurício M. Peixoto and the arrival of Structural Stability to Rio de Janeiro, 1955. [S. l.], 4 de outubro de 2019. Disponível em:

https://arxiv.org/abs/1910.02013v1. Acesso em: 24 de novembro de 2019. 